\title{
Growing research: how neurosurgeons can lead the development of nonsurgical treatments for moyamoya disease
}

\author{
Edward R. Smith, MD \\ Department of Neurosurgery, Boston Children's Hospital, Harvard Medical School, Boston, Massachusetts
}

$\mathrm{M}$ oyamoya disease (MMD) remains a tremendous challenge for both scientists and clinicians. Once defined solely based on radiographic criteria, the understanding of this arteriopathy has progressed over decades. ${ }^{1}$ It is increasingly apparent that there are two related processes - a primary narrowing of specific intracranial vessels, and a secondary response of compensatory collateral circulation development through redirection of blood flow and vasculogenesis. As the sophistication of the field has increased, there has been a trend toward research that focuses on three general areas: diagnosis, prognosis, and therapy. ${ }^{2}$

The diagnosis of MMD has benefited immensely from the recent advances in molecular genetics, with tremendous headway in the identification of specific mutations associated with the classic radiographic and clinical presentations..$^{3-6}$ Prognosis of this condition, with the goal of creating a "crystal ball" to better characterize specific risk factors and help patients predict their future, has been improved with novel imaging methodologies and, increasingly, noninvasive biomarkers. ${ }^{7-11}$ Unfortunately, the third area-therapeutic innovation-has arguably lagged far behind these remarkable diagnostic and prognostic developments. $^{2}$

The case can be made that part of the reason for this dearth of therapeutic research is that many of the clinicians who could identify areas of need and drive this work forward are, sadly, increasingly disconnected from research. Administrative burdens, lack of financial compensation for anything outside of direct clinical care, and growing time demands from things like insurance-related electronic documentation are some of the reasons cited as barriers inhibiting the careers of potential clinicianscientists pursing translational research. Consequently, it is particularly heartening to see work such as the research reported by Dr. Duan and his colleagues in this issue of Neursurgical Focus. ${ }^{12}$
In this study, the team identified a very pragmatic focus for their research: how to improve the growth of surgical collaterals in indirect revascularization for MMD. This unique insight was likely informed by the authors' role as practicing physicians, allowing them to target a defined clinical need. In their paper, they present a simple and elegant hypothesis, summarized as 1) statins, a class of medication typically used for controlling high serum lipid levels, have also been reported to influence the activity of endothelial precursor cells (EPCs) and potentially promote angiogenesis after infarction or stroke; 2) MMD frequently involves ischemia and stroke, with both spontaneous and surgically induced angiogenesis; and 3) therefore, the question arises whether administration of a statin (in this case, atorvastatin) during the perioperative period following indirect revascularization for MMD might help to improve the growth of surgical angiogenesis, as measured by postoperative imaging metrics.

The authors included 106 adult ( $\geq 18$ years of age) patients with a radiographic diagnosis of MMD, and attempting to control for multiple variables (e.g., age, sex, systemic disease, and severity of MMD), ultimately had two well-matched groups of 53 patients each. All patients had indirect revascularization (encephaloduroarteriosynangiosis). One group received $20 \mathrm{mg}$ of atorvastatin daily for 8 weeks after surgery and the other group did not. Taking the medication was voluntary and follow-up occurred at 6 months postoperatively, with the primary metric for comparison being a blinded radiographic score assessing the extent of revascularization territory on catheter angiography. Ultimately, the authors reported that the patients taking the statin had statistically significant increases in the likelihood of demonstrating better postoperative collateralization as measured by the Matsushima grade $(\mathrm{p}=$ 0.006). Of the grade A collaterals, $54 \%$ were on atorvastatin (vs $46 \%$ of the control group), and of the grade B, $\sim 64 \%$ were on atorvastatin (vs $36 \%$ of the control group). 
Conversely, of the poor outcomes with grade C, only $33 \%$ had taken atorvastatin, while the majority of patients experiencing less desirable surgical outcomes (67\%) were not on the statin. All other measures, such as EPC counts, perfusion studies, and clinical outcomes, did not show significant differences between the groups.

As the authors note, there are clearly many potential limitations to this study, some of them quite concerning. The metrics are imprecise, particularly the key result of the Matsushima grade. Patient enrollment was small in number, single-center, and nonrandomized, and the follow-up was incomplete over a relatively short period of time. However, it is crucial to acknowledge that this type of work is absolutely essential in the field of medicine. There is a huge gap between the tidy, controlled environment of in vitro work and the massive cost, time, and effort involved in a multicenter clinical trial. The type of research performed by Dr. Duan and his colleagues serves as a vital bridge between these two extremes that allows knowledge to travel from the benchtop to the bedside.

It is far too easy to play it safe and perform iterative projects - or just to avoid the work involved in translational research altogether. The cost in time and effort is great, the chances of success are low, and the critics are harsh. However, without the willingness to take these risks and persevere in the kind of research presented in this journal, we cannot advance as a field. Like moyamoya vessels, our flow of knowledge will wither and diminish, unless we choose to find new ways of growing the vital lifeblood of translational research.

https://thejns.org/doi/abs/10.3171/2021.6.FOCUS21374

\section{References}

1. Scott RM, Smith ER. Moyamoya disease and moyamoya syndrome. N Engl J Med. 2009;360:1226-1237.

2. Ganesan V, Smith ER. Moyamoya: defining current knowledge gaps. Dev Med Child Neurol. 2015;57(9):786-787.

3. Kundishora AJ, Peters ST, Pinard A, et al. DIAPH1 Variants in non-East Asian patients with sporadic moyamoya disease. JAMA Neurol. Published online June 14, 2021. doi:10.1001/ jamaneurol.2021.1681
4. Pinard A, Guey S, Guo D, et al. The pleiotropy associated with de novo variants in CHD4, CNOT3, and SETD5 extends to moyamoya angiopathy. Genet Med. 2020;22(2):427-431.

5. Milewicz DM, Kwartler CS, Papke CL, Regalado ES, Cao J, Reid AJ. Genetic variants promoting smooth muscle cell proliferation can result in diffuse and diverse vascular diseases: evidence for a hyperplastic vasculomyopathy. Genet Med. 2010;12:196-203.

6. Kamada F, Aoki Y, Narisawa A, et al. A genome-wide association study identifies RNF213 as the first Moyamoya disease gene. J Hum Genet. 2011;56:34-40.

7. Sesen J, Driscoll J, Moses-Gardner A, Orbach DB, Zurakowski D, Smith ER. Non-invasive urinary biomarkers in moyamoya disease. Front Neurol. 2021;12:661952.

8. Pricola Fehnel K, Duggins-Warf M, Zurakowski D, et al. Using urinary bFGF and TIMP3 levels to predict the presence of juvenile pilocytic astrocytoma and establish a distinct biomarker signature. J Neurosurg Pediatr. 2016;18(4):396-407.

9. Smith ER. Moyamoya biomarkers. J Korean Neurosurg Soc. 2015;57(6):415-421.

10. Lee M, Guzman R, Bell-Stephens T, Steinberg GK. Intraoperative blood flow analysis of direct revascularization procedures in patients with moyamoya disease. J Cereb Blood Flow Metab. 2011;31(1):262-274.

11. Elbers J, Armstrong D, Benseler SM, Dlamini N, Steinberg GK, Yeom KW. The utility of collaterals as a biomarker in pediatric unilateral intracranial arteriopathy. Pediatr Neurol. 2018;78:27-34.

12. Wang QN, Bao XY, Zou ZX, et al. The role of atorvastatin in collateral circulation formation induced by encephaloduroarteriosynangiosis: a prospective trial. Neurosurg Focus. 2021; 51(3):E9.

\section{Disclosures}

The author reports no conflict of interest.

\section{Correspondence}

Edward R. Smith: edward.smith@childrens.harvard.edu.

\section{INCLUDE WHEN CITING}

DOI: 10.3171/2021.6.FOCUS21374 